\title{
Un mot du rédacteur
}

Les articles et les notes de pratique du présent numéro de la Revue canadienne dévaluation de programme (RCÉP) mettent en relief la valeur des travaux empiriques pour améliorer les connaissances de la pratique de l'évaluation. Il est gratifiant de voir la quantité et la qualité des travaux d'évaluation envoyés pour publication. Comme à l'habitude pour un numéro régulier de la RCÉP, la présente édition traite d'une vaste gamme de sujets. Nos lecteurs y trouveront au moins un sujet qui contribuera à leurs propres pratique et réflexion.

Si nous commençons par le plus théorique, le premier article de recherche par Suman Budhwani propose un examen critique de l'analyse de contribution, dans un texte qui met en relief certains défis méthodologiques et suggère des adaptations possibles. Jean Marie Buregeya, Astrid Brousselle, Kareen Nour et Christine Loignon utilisent l'analyse de contribution pour évaluer l'utilisation de lévaluation d'impact sur la santé au niveau municipal. Leur article est un exemple parfait de "démantèlement de silos", qui utilise nos connaissances en évaluation pour analyser l'impact d'un outil d'évaluation créé pour le domaine des politiques publiques en santé. Lévaluation de type «advocacy » attire de plus en en plus lattention. Juniper Glass décrit les résultats d'un sondage sur les évaluations de ce type qui visent à influencer les systèmes et les politiques et offre un nouvel éclairage pour orienter les futures évaluations de cette nature. Les deux derniers articles (Veillette-Bourbeau, Otis, Blais, Rousseau et Wainberg et LeClair, Paquette et Letarte) sont des analyses d'implantation qui soulignent le rôle et les défis liés à l'implication des partiesprenantes pour analyser la fidélité de l'implantation.

Trois notes de pratique et de recherche décrivent la façon dont des évaluateurs ont relevé des défis dans des situations particulières dévaluation. Robert K. D. Mclean et ses collègues donnent un aperçu de la conception d'une structure d'évaluation de la gouvernance qui les a aidés à passer outre le défi persistant auquel nous avons tous fait face lorsqu'il est question de produire une évaluation aussi indépendante qu'inclusive. Mettant en lumière un problème sous-jacent dont il est peu question, Vivien Runnels, Caroline Andrew et Jennifer Rae attirent notre attention sur le défi de soutenir l'intérêt porté à lévaluation dans un programme communautaire fondé sur un partenariat, et suggèrent des solutions au problème. Finalement, Ouimet et Morin brossent un tableau de la façon dont l'évaluation peut améliorer les pratiques dorganisations de service social en les rendant plus conformes aux pratiques scientifiques. 
Devant un choix si vaste de sujets, de recherches et d'analyses pertinentes, vous allez certainement survoler plusieurs des articles de ce numéro et vous plonger dans plus d'un. N'oubliez pas que vous pouvez faire part de vos réflexions dans notre forum de discussion en ligne.

Robert Schwartz Rédacteur en chef 
